Canadian Journal of Higher Education

Revue canadienne d'enseignement supérieur

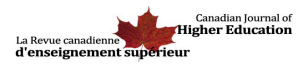

\title{
L'incidence du milieu d'études sur les possibilités de choix de femmes autochtones : typologie de parcours scolaires
}

\author{
Jo-Anni Joncas and Annie Pilote
}

Volume 49, Number 3, 2019

URI: https://id.erudit.org/iderudit/1066635ar

DOI: https://doi.org/10.7202/1066635ar

\section{See table of contents}

Publisher(s)

Canadian Society for the Study of Higher Education

ISSN

2293-6602 (digital)

Explore this journal

Cite this article

Joncas, J.-A. \& Pilote, A. (2019). L'incidence du milieu d'études sur les possibilités de choix de femmes autochtones : typologie de parcours scolaires. Canadian Journal of Higher Education / Revue canadienne d'enseignement supérieur, 49(3), 57-68. https://doi.org/10.7202/1066635ar
Article abstract

This paper aims to assess the impact of the study environment on the opportunities of Indigenous women to complete the school pathway desired. During individual interviews, we collected 19 stories of Indigenous women's experiences at two Quebec universities, one with several initiatives for indigenous students and the other with few. Through a typological analysis, four types of school pathways are presented according to the impact of the study environment; the pathway with: 1) pre-existing opportunities; 2) opportunities highlighted by the study environment; 3) rebound opportunities; and 4) driven by external impulses. Inspired by the capability approach, the results highlight the importance of focusing not only on the services and measures available to these students, but also on their ability to use them to achieve the desired academic path. This requires considering the factors that enable them to make effective use of educational resources for the purpose they choose.
This document is protected by copyright law. Use of the services of Érudit (including reproduction) is subject to its terms and conditions, which can be viewed online.

https://apropos.erudit.org/en/users/policy-on-use/ 


\title{
L'INCIDENCE DU MILIEU D'ÉTUDES SUR LES POSSIBILITÉS DE CHOIX DE FEMMES AUTOCHTONES : TYPOLOGIE DE PARCOURS SCOLAIRES
}

\author{
\begin{tabular}{c|c} 
JO-ANNI JONCAS & ANNIE PILOTE \\
UNIVERSITÉ D'OTTAWA & UNIVERSITÉ LAVAL
\end{tabular}
}

\section{Résumé}

Cet article documente l'incidence du milieu d'études sur les possibilités de femmes autochtones de réaliser le parcours scolaire choisi. Par entretiens individuels, 19 récits de parcours ont été collectés dans deux universités québécoises choisies en fonction de l'importance contrastée des services offerts aux étudiants autochtones. Grâce à une analyse typologique, quatre types de parcours sont définis selon l'effet du milieu d'études : les parcours 1) aux possibilités préexistantes; 2) aux possibilités propulsées par le milieu d'études; 3 ) aux possibilités de rebond et 4) aux possibilités projetées par des impulsions externes. Inspirés par l'approche par les capabilités, les résultats relèvent l'importance de se préoccuper non seulement des services qui sont accessibles à ces étudiantes, mais également de leur capacité à y recourir et à en bénéficier afin de mener le parcours scolaire désiré. Cela demande de prendre en considération les facteurs leur permettant de faire réellement usage des ressources éducatives dans la visée qu'elles choisissent.

Mots-clés : justice sociale, enseignement supérieur, femmes autochtones, typologie

\section{Abstract}

This paper aims to assess the impact of the study environment on the opportunities of Indigenous women to complete the school pathway desired. During individual interviews, we collected 19 stories of Indigenous women's experiences at two Quebec universities, one with several initiatives for indigenous students and the other with few. Through a typological analysis, four types of school pathways are presented according to the impact of the study environment; the pathway with: 1) pre-existing opportunities; 2) opportunities highlighted by the study environment; 3) rebound opportunities; and 4) driven by external impulses. Inspired by the capability approach, the results highlight the importance of focusing not only on the services and measures available to these students, but also on their ability to use them to achieve the desired academic path. This requires considering the factors that enable them to make effective use of educational resources for the purpose they choose.

Keywords: social justice, higher education, Indigenous women, typology

\section{Introduction}

II y a plus de vingt ans, la Commission royale sur les peuples autochtones (CRPA) (1996) a levé le voile sur les conditions socioéconomiques préoccupantes des Premiers peuples ${ }^{1}$ au Canada. Encore aujourd'hui, des communautés autochtones n'ont pas accès à l'eau potable, à l'électricité, au chauffage et à des logements décents (Anaya, 2014). Comment expliquer une telle situation dans un pays dont le niveau de vie enviable est clamé haut et fort? Nous ne pouvons faire autrement que de nous interroger sur l'efficacité des programmes, services et mesures destinés aux Premiers peuples
(Lévesque, 2015). En 2015, les appels à l'action de la Commission de vérité et réconciliation (CVR) exprimaient formellement les demandes des Premiers peuples à l'égard des gouvernements du pays afin d'obtenir de meilleures conditions de vie. La recommandation numéro sept indique : " Nous demandons au gouvernement fédéral d'élaborer, de concert avec les groupes autochtones, une stratégie conjointe pour combler les écarts en matière d'éducation et d'emploi entre les Canadiens autochtones et les Canadiens non autochtones » (p. 2).

Cette article s'inspire d'une recherche qui porte sur la justice sociale à l'enseignement supérieur pour les 
femmes autochtones. II présente une partie des résultats tirés d'une analyse typologique du récit de parcours de 19 femmes autochtones universitaires. Ceux-ci relèvent l'importance de se préoccuper non seulement des services (incluant les politiques, programmes et mesures) qui sont accessibles à ces étudiantes, mais également de leur capacité à y recourir et à en bénéficier dans l'optique de mener le parcours scolaire qu'elles désirent.

\section{Recension des écrits : un problème d'égalité}

Depuis le début de la colonisation, les Premiers peuples évoluent dans des systèmes scolaires qui les oppressent (CRPA, 1996; CVR, 2015). Leurs luttes pour l'autonomie de gouvernance témoignent de leur volonté de reprendre en main ce lieu de transfert idéologique et de savoirs. Or, les taux de scolarisation des Premiers peuples restent faibles comparativement à ceux des populations allochtones, et ce, à tous les niveaux d'études (Statistique Canada, 2016a). Une proportion relativement faible d'élèves autochtones obtiennent un diplôme d'études secondaires. Dans ce contexte, il n'est guère surprenant qu'ils soient sous-représentés à tous les autres niveaux d'études (excluant la formation professionnelle), et particulièrement au niveau universitaire si l'on compare aux études collégiales. En 2016, 5 \% des membres des Premières Nations " inscrits " vivant en communauté, $11 \%$ des membres des Premières Nations " inscrits " vivant hors communauté, $2 \%$ des Inuits du Nunangat, $12 \%$ des Inuits à l'extérieur du Nunangat et $14 \%$ des Métis avaient un diplôme universitaire, comparativement à $29 \%$ de la population allochtone (Statistique Canada, 2016a).

Le problème de la faible scolarisation universitaire des Premiers peuples au pays est de plus en plus documenté. Trois courants explicatifs, bien que non mutuellement exclusifs, sont privilégiés. Un premier explique la situation par les facteurs de persévérance scolaire des Autochtones (Adelman et al., 2010; Aragon, 2002; Huffman, 2001; Larimore et McClellan, 2005; Montgomery et al., 2000; Whitley, 2014). Ces facteurs se concentrent sur les caractéristiques individuelles et culturelles des étudiants autochtones pour expliquer les inégalités scolaires. Ces études ont permis de mettre en lumière plusieurs facteurs qui nuiraient à la persévérance scolaire des étudiants autochtones, par exemple, un style d'apprentissage différent. Elles ont aussi documenté des facteurs qui faciliteraient leur persévérance, comme la fierté identitaire.

Un deuxième courant s'intéresse davantage à l'expérience scolaire des étudiants autochtones (Andrade, 2014; Fox, 2013; Joncas, 2013; Mosholder et al., 2011; Ratel, 2017; Rodon, 2008; Sonn et al., 2000). La plupart de ces études tentent de comprendre comment les étudiants autochtones donnent sens à leur expérience scolaire et s'adaptent aux exigences et aux attentes de leur institution scolaire. Elles s'intéressent aussi aux relations qu'entretiennent les étudiants autochtones avec les autres acteurs du milieu scolaire. Par exemple, elles ont démontré que la signification de la réussite scolaire serait davantage attribuée au développement personnel et à l'acquisition de compétences qu'à la diplomation. Elles ont mis en exergue le choc culturel vécu par des étudiants autochtones dans des institutions postsecondaires canadiennes.

Un troisième courant se penche sur l'histoire, les structures et les systèmes scolaires pour justifier le problème (Alfred, 2004; Bailey, 2015; Battiste, 2013; Kerr, 2014; Lavell Harvard, 2011; Mihesuah, 2004; Pidgeon, Archibald et Hawkey, 2014; Rasmussen, 2001; Timmons, 2009). Entre autres, ces études expliquent les faibles taux de scolarisation à l'université par l'eurocentrisme, la persistance de pratiques coloniales et les structures (néo)coloniales oppressives des systèmes d'éducation qui discriminent les étudiants autochtones.

Malgré l'éclairage que ces écrits apportent sur l'influence de l'identité autochtone, du sens de l'expérience scolaire et des discriminations systémiques pour expliquer la faible scolarisation des étudiants universitaires autochtones, il semble pertinent de pousser la réflexion en adoptant une analyse multidimensionnelle qui s'intéresse aux relations entre l'individu, le sens donné à son parcours, ses relations sociales et l'influence du contexte d'études.

Par ailleurs, peu de recherches s'intéressent spécifiquement aux femmes autochtones dans les études supérieures au Canada (Lavell Harvard, 2011; Lévesque et Labrecque, 2007; Martin, 2001; Neeganagwedgin, 2011) et aux États-Unis (Andrade, 2014; Arbor, 2008; Bamdas, 2009; Castagno, 2005; Fox, 2013; Jaime 2003; Sankhulani, 2007; Waterman et Lindley, 2013). Ces recherches ont mis en évidence le besoin d'étudier séparément le parcours scolaire 
des femmes autochtones en raison des différences relevées entre les parcours de femmes et d'hommes autochtones, mais aussi entre les parcours de femmes autochtones et allochtones. Par exemple, les femmes autochtones sont plus nombreuses à l'université que les hommes (Statistique Canada, 2016), ont un nombre élevé de grossesses à l'adolescence (Joncas et Roy, 2016), sont plus souvent des mères à l'université que les étudiantes allochtones (Statistique Canada, 2016b) et sont nombreuses en situation de monoparentalité (Guimond et al., 2012). Sankhulani (2007) souligne que les hommes autochtones peuvent trouver de l'emploi et obtenir des promotions plus facilement que les femmes autochtones. Ils ont aussi un revenu médian plus élevé, bien qu'ils soient moins instruits (Statistique Canada, 2016b). C'est pourquoi nous avons choisi d'analyser le problème de la faible scolarisation universitaire chez les Premiers peuples en nous centrant sur l'expérience de femmes.

L'objectif principal de la recherche est d'analyser comment le milieu d'études de femmes autochtones à l'université contribue au développement de leurs possibilités de choisir et de réaliser le parcours scolaire qu'elles valorisent. Par " milieu d'études ", il faut comprendre tant les lois, politiques et règlements, systèmes scolaires et établissements d'enseignement que les relations des étudiants durant leurs études.

\section{Assises théoriques : approche par les capabilités}

Tous les choix en faveur de l'égalité scolaire dépendent d'une conception particulière de l'égalité. La plupart de ces conceptions se concentrent sur une seule variable, comme les ressources (égalité des places et des droits), le traitement (égalité des chances) ou les résultats (égalité de résultats) (Dubet, 2010; Potvin, 2014; Verhoeven, Orianne et Dupriez, 2007). Or, un système scolaire pleinement juste devrait prendre en considération non seulement les ressources et les traitements octroyés, mais également les réalisations des apprenants (Verhoeven et al., 2007). Cela demande de tenir compte des facteurs permettant à un apprenant de faire réellement usage des ressources éducatives dans la visée qu'il choisit. Cette conception de l'égalité cadre avec l'approche par les capabilités d'Armatya Sen (2010).
Selon Sen, il est nécessaire de prendre appui sur les ressources dont les individus disposent, mais principalement sur leur capacité à utiliser ces ressources pour augmenter leurs possibilités de choix. En contexte scolaire, c'est dire que l'éducation devrait travailler à augmenter l'étendue des choix des apprenants en travaillant sur leurs possibilités d'utiliser les ressources pour les convertir en libertés de choix (Verhoeven et al., 2007). L'éducation doit donc s'assurer que les apprenants ont accès aux mêmes ressources et qu'ils ont les mêmes possibilités de les utiliser, ce qui les mène vers des choix éducationnels équivalents (Verhoeven et al., 2007). Pour y parvenir, les services se concentrent sur les besoins des apprenants pour ajuster les ressources et pour repérer les obstacles qui les empêchent d'accéder au plus vaste éventail de choix possible (Conseil supérieur de l'éducation, 2016; Potvin, 2014).

Sen (2000) a développé un cadre compréhensif qui se fonde sur différents concepts pour opérationnaliser celui de justice. Les concepts mobilisés dans son étude sont: 1) les ressources et droits formels; 2) les capabilités; 3) les facteurs de conversion; et 4) les fonctionnements. Les ressources et droits formels représentent les biens, les services et les droits dont dispose un individu pour accéder à la justice. Les facteurs de conversion (FC), concept central de l'approche, influencent la capacité d'un individu à convertir une ressource en une liberté de choisir la vie qu'il a des raisons de valoriser. Ils peuvent être personnels, socioculturels ou environnementaux. Ces facteurs font varier positivement $(\mathrm{FC}+)$ ou négativement (FC-) les possibilités de choix. Par exemple, Sen (2000) souligne qu'il y a des écarts systématiques entre les libertés dont jouissent respectivement les femmes et les hommes au sein de diverses sociétés dans l'utilisation des revenus (ressources). Les femmes peuvent souffrir de discrimination (FC- environnementaux) quand vient le temps de convertir leur revenu en réalisations particulières. L'accent mis sur les FC permet de mettre en évidence les possibilités inégales de conversion des ressources en libertés de choisir. Les capabilités (de l'anglais capabalities) sont les possibilités réelles de choix dont dispose un individu d'u être " et de " faire " ce qu'il a des raisons de valoriser. Les fonctionnements sont les possibilités de choix mises en œuvre par les individus. Nous avons schématisé l'approche par les capabilités dans la figure suivante (Figure 1). Globalement, lorsque les ressources sont disponibles, si l'influence des FC+ l'emporte sur les FC-, l'individu 
est mené vers une possibilité réelle de choix qui peut déboucher sur un fonctionnement. Dans le cas inverse, les FC- débouchent sur un éventail de choix plus limité, duquel pourraient résulter un fonctionnement contraint ou une absence de fonctionnement. Ces réalisations peuvent être converties en ressources pour atteindre d'autres possibilités, comme c'est le cas, par exemple, pour un diplôme d'études.

\section{Assises méthodologiques}

Les choix méthodologiques ont pris en compte les besoins et les intérêts des femmes autochtones exprimés notamment dans des écrits antérieurs. L'étude multicas est la stratégie de recherche qui a été retenue, car elle permet notamment d'étudier en profondeur un phénomène, tout en tenant compte du contexte et des

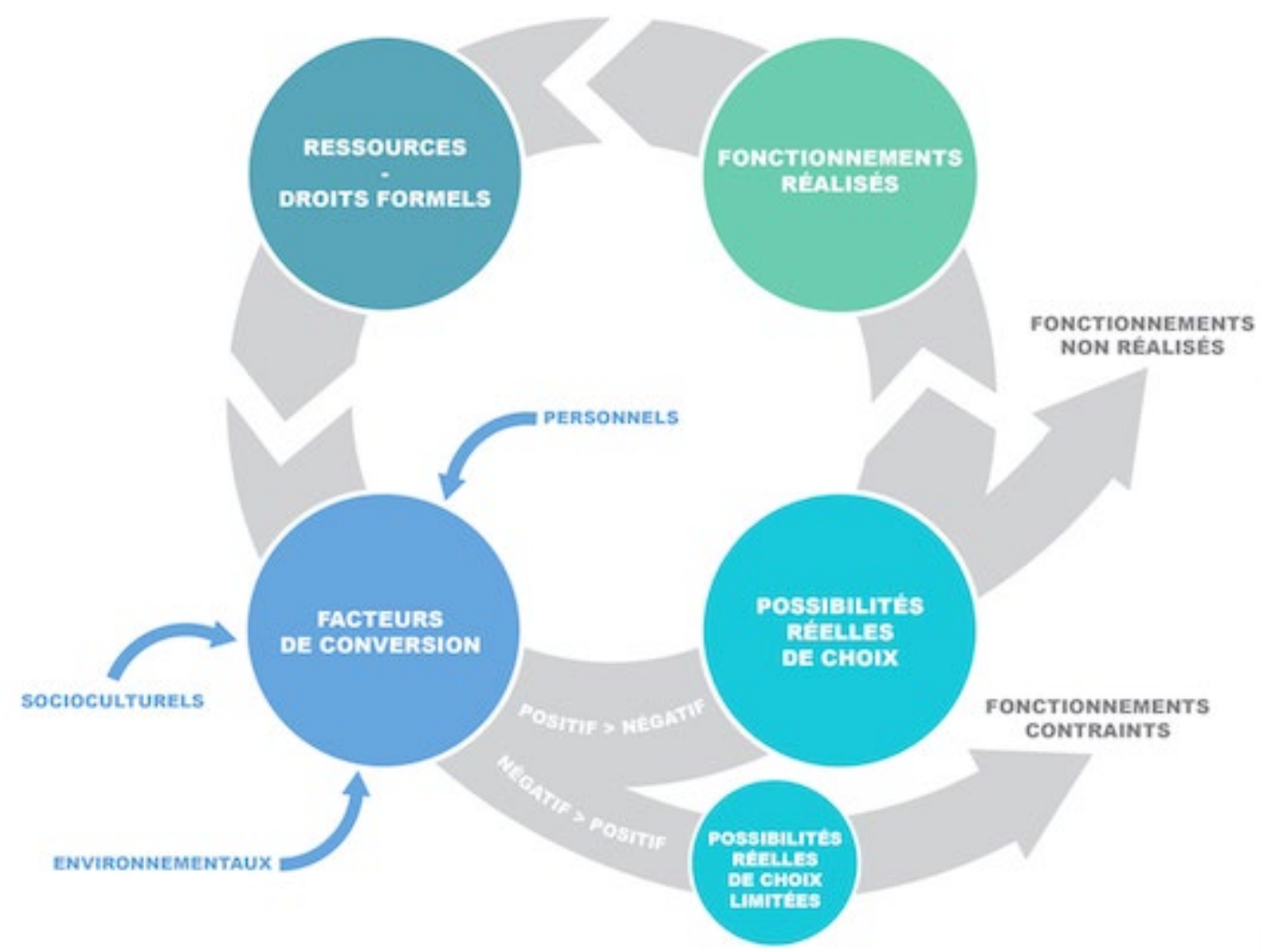

Figure 1. Schématisation de l'approche par les capabilités

L'approche par les capabilités va donc au-delà d'une théorie purement axée sur la distribution de ressources en s'assurant qu'il existe des chances réelles pour l'ensemble des individus de saisir ces ressources et de les convertir en possibilités de choisir ce qui a de l'importance pour eux. Dans le cadre de cette recherche, cette approche a permis d'analyser les possibilités pour des femmes autochtones d'effectuer le parcours scolaire choisi. nombreuses variables susceptibles de révéler la relation entre l'individu et les structures (Yin, 2012). Les cas sont deux universités québécoises, UnivA et UnivB, choisies en fonction de l'importance contrastée des services qu'elles offrent pour favoriser l'expérience éducative des étudiants autochtones. Même si chacune des universités accueille une bonne part des étudiants autochtones du Québec, I'UnivA, qui est l'une des grandes universités de la province, située en milieu urbain, n'a pas d'énoncé clair sur sa position à l'égard des étudiants autochtones et n'a pas de services destinés spécifiquement à ce groupe, outre une association étudiante autochtone 
qui est tenue par des étudiants autochtones grâce au soutien d'un centre d'études. Les nombreux services qui y sont offerts sont inclusifs et ouverts à tous les étudiants, à l'exception de quelques services adaptés aux spécificités des étudiants internationaux ou des étudiants présentant un handicap. L'UnivB, plus petite et située en région éloignée, démontre une volonté institutionnelle relativement aux étudiants autochtones, qui sont au cœur des services. Le choix de travailler avec seulement deux cas contrastés présente certains avantages pour la compréhension de l'incidence du milieu d'études sur les possibilités de choix des femmes autochtones, mais limite la portée de la comparaison tant les cas sont différents.

Dans chacune des universités, nous avons fait une monographie, des entretiens individuels avec des membres du personnel et des entretiens sous forme de récit de parcours avec des étudiantes autochtones. Seuls les récits des 19 étudiantes participantes (8 à l'UnivA et 11 à l'UnivB) sont mobilisés dans cet article. Trois critères ont guidé leur sélection : 1) s'identifier en tant que femme; 2) s'identifier comme Autochtone; et 3) être étudiante à l'UnivA ou l'UnivB. Soulignons que 16 des 19 participantes étaient enceintes ou avaient au moins un enfant. Leur recrutement s'est effectué par des affiches, des messages sur les médias sociaux et/ou dans des bulletins d'information et, lorsque possible, l'envoi de courriels. La collecte de données s'est déroulée dans l'année scolaire 2015-2016. Les entretiens ont duré environ une heure. Une partenaire de recherche, qui était étudiante autochtone au premier cycle universitaire, a participé au projet en prenant part à l'élaboration des outils de collecte, à la collecte et à l'analyse de données. Elle a notamment mené les entretiens avec les femmes autochtones.

L'étude comporte quatre niveaux d'analyse des données (macrosocial, mésocial, microsocial et transversal). Dans cet article, nous nous concentrons sur l'analyse typologique du récit du parcours scolaire des étudiantes (Schnapper, 2012). L'analyse met en exergue leur liberté de choisir et de réaliser le parcours envisagé en prenant en compte les mouvements institutionnels (Bonvin, 2014). Une importance a été accordée à la variabilité des parcours dans le temps, aux transactions entre les diverses sphères de la vie et aux contextes relationnels, sociaux et institutionnels qui ont un effet sur le parcours scolaire des participantes. Afin de réaliser cette typologie, une fiche synthèse du récit de chaque participante a été créée en mettant l'accent sur les moments charnières. Les fiches sont construites de manière diachronique afin de reconstruire la trame temporelle des parcours.

\section{Typologie de parcours}

Quatre types de parcours ont été dégagés du corpus selon l'incidence du milieu d'études des répondantes sur leurs possibilités de choix grâce à la méthode par " tas " (Schnapper, 2012). Ces types ne sont pas liés à des individus concrets, mais à des expériences sociales (Schnapper, 2012). Ils ne constituent pas la représentation exacte des récits des participantes, mais une abstraction de leurs caractéristiques et de leur rapport au contexte social. Même si certains des parcours se " classent " systématiquement dans tel ou tel type, d'autres se trouvent à la frontière entre différents types.

\section{Le parcours scolaire aux possibilités préexistantes}

Un premier type, nommé " parcours scolaire aux possibilités préexistantes ", montre que le milieu d'études a une faible incidence sur les possibilités des étudiantes de réaliser le parcours scolaire choisi. C'est le cas pour huit des 19 participantes. Leur parcours est généralement linéaire, avec peu d'allers et retours entre les programmes d'études et les établissements d'enseignement. Certaines ont mis leurs études sur pause pour travailler ou pour s'occuper de leurs enfants. Elles ont toutes obtenu un diplôme d'études secondaires (DES) et collégiales (DEC) avant d'entreprendre des études universitaires (fonctionnements/ressources). Les compétences et connaissances développées au cours des études collégiales (FC+) leur permettent notamment de profiter pleinement de leur programme universitaire. Le désir d'accéder à un diplôme universitaire s'explique par une raison précise $(\mathrm{FC}+)$ qui donne un sens à leurs études. Par exemple, une répondante a choisi de faire un certificat même si elle avait un emploi apprécié dans sa communauté dans le souhait de sortir de sa routine, d'approfondir ses connaissances et de montrer l'exemple à ses enfants. Une autre explique que son choix d'études la mènera vers une profession qui lui permettra de nourrir son appétit pour les sciences et la relation d'aide 
tout en lui permettant d'œuvrer dans les communautés autochtones.

Ce type de parcours scolaire se caractérise également par le fait que les étudiantes disposent de plusieurs ressources de départ (bourses d'études, revenus autres, voiture, etc.) qu'elles ont pu transformer en possibilités d'être et de faire notamment grâce à des $\mathrm{FC}+$ (services du centre d'amitié autochtone, soutien des proches, etc.). Ces participantes sont alors arrivées à l'université bien outillées en matière de ressources et de $\mathrm{FC}+$ principalement personnels et socioculturels. Elles ont rencontré peu de problèmes majeurs et elles sont conscientes de l'utilité de leurs études universitaires. Leurs possibilités de réussir le parcours scolaire désiré sont donc élevées et leur milieu d'études a peu d'effet sur ces possibilités.

\section{Le parcours scolaire aux possibilités propulsées par le milieu d'études}

Le second type renvoie au " parcours scolaire aux possibilités propulsées par le milieu d'études ", dans lequel les possibilités de choix des étudiantes sont très influencées parleur milieu d'études. C'estle cas pour quatre participantes. Leur parcours est parsemé de pauses, de bifurcations et d'embûches. Trois ont leur DES, deux ont un DEC et une détient une attestation d'études collégiales (fonctionnements/ressources). L'entrée à l'université s'est faite après plusieurs années à l'extérieur des murs de l'école. Leur parcours laissait peu présager un passage à l'université tant les FC- (relations problématiques, santé mentale fragile, etc.) sont prépondérants par rapport aux $\mathrm{FC}+$. Le motif du retour aux études est principalement lié au désir d'apporter des changements positifs à une situation difficile. Par exemple, une étudiante a entrepris des études universitaires afin d'améliorer sa situation problématique sur les plans professionnel, relationnel et mental. Elle a quitté sa communauté avec son jeune enfant pour s'installer près de son université. Avec l'aide de services communautaires ( $\left.F C_{+}\right)$, elle a réorganisé sa vie. Même si ses premières sessions à l'université ont été exigeantes, elle s'est accrochée grâce aux ressources disponibles (bourses, logement, etc.), mais aussi au soutien de ses collègues, de ses proches et du personnel universitaire (FC+).

Les étudiantes qui ont ce type de parcours comptent sur plusieurs ressources (surtout communautaires, comme un centre d'hébergement pour femmes) conjuguées à des FC+ de leur milieu d'études pour ouvrir leurs possibilités de choix, et ce, même si leur parcours est marqué de FC- très influents. Par exemple, l'une d'entre elles justifie sa réussite scolaire par les services universitaires (FC+) auxquels elle a accès et qu'elle utilise, tels que l'aide pédagogique, les associations étudiantes, de même que le soutien du personnel universitaire, de ses collègues autochtones et d'une mentore (FC+). Elle ajoute que sa participation à un programme préparatoire $(F C+)$ lui a permis d'apprendre le métier d'étudiant universitaire. Les quatre étudiantes qui ont ce type de parcours sont toutes originaires d'une communauté autochtone très éloignée de leur université (FC-). Loin de leurs proches, ces étudiantes reconnaissent l'apport indéniable de leur " famille-école » (FC+).

II en résulte que même si les répondantes qui ont ce type de parcours ont rencontré de nombreux obstacles (FC-) et que leur accès et leur réussite aux études universitaires pouvaient sembler, à première vue, improbables, leur récit montre comment des FC+ les ont propulsées. Ces FC+ sont pour la plupart environnementaux, issus de leur milieu d'études. Ces femmes ont dorénavant des possibilités favorables pour réussir le parcours choisi.

\section{Le parcours scolaire aux possibilités de rebond}

Le troisième type, le " parcours scolaire aux possibilités de rebond ", concerne un parcours où le milieu d'études a une incidence plus ou moins notable sur les possibilités des répondantes de réaliser le parcours scolaire souhaité. Il se caractérise par le fait que les études postsecondaires semblent être la seule issue possible après les études secondaires, tant la pression sociale est forte (FC-). Cette pression sociale provient principalement des proches, qui projettent des aspirations scolaires élevées. C'est le cas pour trois étudiantes qui ont pris la décision de poursuivre leurs études postsecondaires après l'obtention de leur DES (fonctionnements/ressources) sans trop connaître leurs intentions de départ (FC-). Le but de leurs études s'est construit tout au long de leurs études. Par exemple, les parents d'une répondante lui ont fait comprendre très tôt dans sa vie que si elle voulait subvenir à ses besoins, elle ne pourrait plus se fier au territoire comme eux l'ont fait étant donné la dégradation de l'environnement. Cela 
passerait par une bonne éducation. Elle a décidé de faire des études collégiales dans un programme général, car elle ne savait pas quoi faire. Entre ses études collégiales et universitaires, elle a eu un enfant qui n'était pas prévu. Elle a par la suite fait un premier baccalauréat, qui lui a pris près de 10 ans.

La première phase des études postsecondaires de ces répondantes est plus exploratoire, parsemée de périodes de remise en question (FC-). De plus, ces étudiantes ont vécu divers problèmes personnels qui sont venus brouiller les cartes (FC-). Malgré les embûches, cette phase leur a permis d'explorer différents programmes, a confirmé leurs aspirations $(\mathrm{FC}+)$, a solidifié leur identité ( $\mathrm{FC}+$ ) et les a menées vers leur $\mathrm{DEC}$ (fonctionnements/ressources).

La deuxième phase survient à l'université. Le choix d'études des étudiantes étant déterminé et significatif $(\mathrm{FC}+)$, leur parcours se stabilise. Ces étudiantes détiennent alors la plupart des outils nécessaires pour réussir le programme désiré. Ces outils, comme leurs habiletés organisationnelles ou linguistiques (FC+), ont notamment été développés durant leurs études collégiales. C'est probablement ce qui explique pourquoi ces participantes utilisent très peu les services de leur université.

Autrement dit, ce type de parcours se caractérise par une phase exploratoire suivie d'une phase fixée. Plus long que la moyenne, ce parcours leur a permis de développer leur maturité et leur connaissance d'ellesmêmes. Par les détours empruntés, ces étudiantes ont développé leurs propres FC+. Une fois à l'université, elles ressentent peu le besoin de s'appuyer sur les services disponibles. Leurs possibilités de réussir le parcours scolaire désiré sont donc passées de faibles à assez fortes.

\section{Le parcours scolaire aux possibilités projetées par des impulsions externes}

Dans le type du " parcours scolaire aux possibilités projetées par des impulsions externes ", le milieu d'études a une incidence assez forte sur les possibilités des répondantes de faire le parcours scolaire souhaité. Ce type de parcours prévaut pour quatre étudiantes, qui ont toutes un DEC (trois ont leur DES) (fonctionnements/ ressources). Leurs études universitaires sont perçues comme un moyen pour arriver à une autre fin qu'un diplôme (FC-). Par exemple, une étudiante a décidé de faire des études universitaires, car elle s'ennuyait dans son emploi. Elle qui n'était d'ailleurs pas certaine d'aller à l'université s'est décidée lorsque des amis lui ont fait remarquer qu'elle avait de la chance d'avoir accès à un soutien financier de sa communauté (ressource). Elle mentionne qu'en tant que mère monoparentale (FC-), ce diplôme universitaire lui permettra de devenir indépendante financièrement et d'assurer une bonne vie à son enfant (FC+). Une autre étudiante précise que son retour aux études a été motivé par ses enfants, afin qu'ils puissent bénéficier de la qualité de l'éducation disponible dans une ville universitaire en comparaison avec celle offerte dans sa communauté (FC+).

Les étudiantes qui s'inscrivent dans ce type de parcours scolaire ont une motivation scolaire plus ou moins stable (FC-). Cela semble logique lorsque nous nous référons aux raisons externes qui les ont poussées à s'inscrire à l'université. Cela dit, elles détiennent des FC+ qui sont d'un grand apport : agente de relations humaines de l'université, association étudiante autochtone, service de soutien pédagogique, cours de dépannage, etc. Ainsi, leur milieu d'études (que ce soit les collègues, le personnel, les services, etc.) a un impact positif sur leur expérience d'études universitaires, particulièrement en soutenant leur persévérance scolaire lorsqu'elles manquent de motivation (FC+).

Les possibilités de réaliser le parcours scolaire souhaité des étudiantes qui s'inscrivent dans ce type de parcours sont assez précaires. Les raisons du retour aux études sont motivées par les autres et leur réussite repose souvent sur une motivation fluctuante. Leurs possibilités s'en trouvent alors fragilisées, accentuant l'importance du soutien du milieu d'études.

\section{Discussion : une question de con- version}

Le Tableau 1 illustre le type de parcours selon l'université d'attache de chacune des répondantes et l'incidence du milieu d'études sur les possibilités. 
Tableau 1. Types de parcours scolaire selon l'université d'attache

\begin{tabular}{|c|c|c|c|c|c|}
\hline$\searrow_{\begin{array}{r}\text { Type } \\
\text { Incidence }\end{array}}$ & $\begin{array}{c}\text { 1. Aux possibil- } \\
\text { ités préexistan- } \\
\text { tes } \\
\text { Faible }\end{array}$ & $\begin{array}{l}\text { 2. Aux possibilités } \\
\text { propulsées par le } \\
\text { milieu d'études } \\
\text { Forte }\end{array}$ & $\begin{array}{l}\text { 3. Aux possibil- } \\
\text { ités de rebond } \\
\text { Assez faible }\end{array}$ & $\begin{array}{l}\text { 4. Aux possibil- } \\
\text { ités projetées } \\
\text { par des impul- } \\
\text { sions externes } \\
\text { Assez forte }\end{array}$ & Total \\
\hline $\begin{array}{l}\text { Étudiantes de } \\
\text { l'Université A }\end{array}$ & 5 & - & 2 & 1 & 8 \\
\hline $\begin{array}{l}\text { Étudiantes de } \\
\text { l'Université B }\end{array}$ & 3 & 4 & 1 & 3 & 11 \\
\hline Total & 8 & 4 & 3 & 4 & 19 \\
\hline
\end{tabular}

Les parcours peuvent se classer en deux groupes, selon l'influence des FC des milieux d'études sur les possibilités de choix des répondantes. II s'agit 1) des parcours où le milieu d'études, par des FC environnementaux, a eu une incidence considérable; et 2) des parcours qui se caractérisent par une incidence moins importante du milieu d'études.

Huit des 19 répondantes qui ont un type de parcours scolaire aux possibilités propulsées ou projetées s'inscrivent dans le premier groupe. Sept étudientà l'UnivB et une à l'UnivA. Pour plusieurs, leur milieu d'études est une condition de la réalisation du parcours scolaire choisi. Par exemple, une répondante qui a un parcours aux possibilités propulsées croit que sans les mesures de soutien de l'UnivB, il serait difficile de poursuivre ses études. Les analyses confirment que les efforts de I'UnivB pour soutenir les étudiantes autochtones ont des retombées positives, notamment chez les étudiantes avec des possibilités de départ plus précaires. Lorsque la volonté institutionnelle est présente et que des actions sont déployées en ce sens, les universités peuvent soutenir des étudiantes autochtones en position précaire de réussir le programme scolaire espéré en prenant réellement en compte leurs spécificités. Cela requiert, comme relevé dans plusieurs études, de considérer les effets de l'eurocentrisme et de l'historique colonial des systèmes scolaires sur les possibilités des étudiantes en reconnaissant davantage les Premiers peuples et leurs savoirs dans les structures scolaires, entre autres (Alfred, 2004; Kerr, 2014; Battiste, 2013; Mihesuah, 2004; Pidgeon et al., 2014; Timmons, 2009).
Onze des 19 répondantes ont un parcours qui correspond au second groupe. Sept d'entre elles étudient à l'UnivA et quatre à l'UnivB. Leur parcours présente des FC+ personnels (p. ex. : aspirations précises, compétences et connaissances, ancrage identitaire, maîtrise de la langue d'enseignement) et des FC+ socioculturels (p. ex. : soutien familial et extrafamilial, etc.) très importants comparativement aux FC environnementaux issus du milieu d'études. Ainsi, ces étudiantes persévèrent grâce à des FC individuels et socioculturels marqués. Cela rejoint les constats de plusieurs études s'intéressant aux facteurs de réussite scolaire des étudiants autochtones (Adelman et al., 2010; Aragon, 2002; Larimore et McClellan, 2005; Montgomery et al., 2000; Whitley, 2014) et d'études qui se penchent sur le sens de l'expérience scolaire des étudiants autochtones (Fox, 2013; Joncas, 2013; Mosholder et al., 2011; Ratel, 2017; Rodon, 2008; Sonn et al., 2000).

À la lumière des récits des répondantes, nous voyons que celles qui étudient à l'UnivB utilisent davantage les services en place, en grande partie grâce aux FC environnementaux. Ces mesures qui reconnaissent les spécificités des étudiantes autochtones semblent favorables pour permettre à chacune de développer son plein potentiel, particulièrement pour celles dont les ressources de départ étaient plus limitées. Au-delà du fait que l'UnivA offre une panoplie de services à sa communauté étudiante, les femmes autochtones rencontrées semblent peu les utiliser. Cela peut s'expliquer parce qu'elles n'en ont pas besoin ou qu'elles se tournent vers leurs proches en cas de besoin, entre 
autres. Cependant, certains témoignages d'étudiantes de l'UnivA qui s'inscrivent dans des parcours où le milieu d'études a peu d'influence suggèrent qu'elles ne font pas appel à ces services, car ils ne sont pas adaptés à leurs besoins. Par exemple, une participante explique que si elle avait besoin d'aide dans ses études, elle se débrouillerait seule. De plus, une majorité des étudiantes de l'UnivA ont eu de la difficulté à s'adapter à la vie d'étudiante universitaire, plusieurs ont vécu des épisodes de solitude, quelques-unes ont eu une intégration sociale difficile à l'université, certaines ont vécu des discriminations de professeurs, de professionnels universitaires et d'autres étudiantes, etc. Une étudiante de l'UnivA mentionne : " [...] j'ai fait un témoignage sur les Autochtones et là, je suis rendue l'Autochtone de service dans ce cours-là. [...] On n'a pas les opportunités d'être reconnues plus que cela à l'Université ". II faut donc comprendre que même si ces étudiantes ont un type de parcours où le milieu d'études a peu d'incidence, ce n'est pas parce qu'elles n'ont pas de besoins. D'ailleurs, nombreuses sont les étudiantes de l'UnivA qui se tournent vers leurs proches en cas de problèmes.

Ce n'est pas tout de mettre des ressources en place, encore faut-il veiller à ce que les étudiants aient la possibilité de les utiliser et dans l'affirmative, qu'elles ouvrent aux étudiants de réelles libertés de choix qui ont du sens pour eux, notamment pour les étudiants les plus discriminés, comme les femmes autochtones (Conseil supérieur de l'éducation, 2016; Potvin, 2014; Sen, 2000, 2010). Les propos de ces étudiantes nous rappellent les résultats de l'étude de Lavell-Harvard (2011), qui suggèrent que les étudiantes autochtones qui luttent pour s'intégrer (comme c'est le cas pour six de nos répondantes de l'UnivA) pourraient avoir de la réticence à demander de l'aide, craignant que cela ne confirme les croyances de certains qui accusent les étudiants autochtones de réussir grâce à des " accès spéciaux » à certains programmes d'études pour les individus autochtones et de prendre les places qui seraient autrement occupées par des étudiants allochtones. Mihesuah (2004) avance qu'à l'université, des étudiants autochtones peuvent ne pas utiliser des services s'ils sont donnés par un intervenant allochtone en raison de son manque de reconnaissance et de respect face aux cultures autochtones. Ces étudiants ne seraient pas préparés à vivre cet inconfort culturel. Comme relevé dans d'autres études, les répondantes qui persévèrent à l'UnivA ont besoin d'un fort ancrage identitaire pour circuler dans un monde où elles sont minoritaires et où les cultures autochtones sont peu prises en compte (Huffman, 2001; Joncas, 2013; Montgomery et al., 2000).

Au final, le rapport de la CVR (2015) a clairement indiqué que les universités canadiennes ont un rôle fondamental à jouer dans la lutte contre les inégalités auprès des prochaines générations de Canadiens. Ce rôle s'étend au-delà des statistiques alarmantes à propos de l'éducation autochtone, et concerne notamment le processus de réconciliation de la société canadienne (CRV, 2015). Même si les parcours scolaires des femmes autochtones sont loin d'être identiques, il n'en demeure pas moins que les universités ont une responsabilité sociohistorique. Par ailleurs, la présence de services pour favoriser les possibilités de choix des étudiantes autochtones à l'université n'est pas suffisante à elle seule. Soulignons, entre autres, l'importance de la famille et du bien-être dans le développement des possibilités des étudiantes rencontrées. Nous ne pouvons pas passer sous silence l'influence principalement positive qu'ont les enfants des répondantes $\left(F C_{+}\right)$sur leur possibilité de réaliser leur parcours. Souvent, comme relevé dans d'autres études, il s'agit de la motivation du retour aux études et du principal facteur de persévérance, et ce, même si parfois la conciliation études-famille est laborieuse (FC-) (Andrade, 2014; Lavell-Harvard, 2011; Neeganagwedgin, 2011).

\section{Conclusion}

Les études universitaires sont importantes et leur pertinence n'est plus à démontrer, notamment en raison de leur influence sur les possibilités de choix des femmes autochtones. II est plus que nécessaire de leur assurer la réussite du parcours scolaire qu'elles ont choisi, car l'impact positif qu'elles auront sur leur famille, leur communauté et la société est fort significatif. Encore trop peu nombreuses à l'université, notamment au Québec, il import de veiller à leur recrutement, mais aussi à la réalisation du parcours de celles qui y parviennent.

Nous avons souhaité montrer en quoi les universités peuvent contribuer aux possibilités de choix de femmes autochtones. Les résultats de l'analyse typologique du récit du parcours scolaire de 19 femmes autochtones universitaires ont mis en évidence quatre types de parcours selon l'influence du milieu scolaire sur les possibilités de réaliser le parcours scolaire désiré. En 
nous référant à l'université d'attache des répondantes, nous avons fait la réflexion que lorsque la volonté institutionnelle est présente et que des actions sont déployées en ce sens par l'instauration de FC environnementaux, les universités peuvent permettre à des individus ayant un parcours scolaire plus déviant de la norme de réussir le programme choisi. Ces analyses nous mènent à la conclusion selon laquelle il est important de se préoccuper non seulement des ressources qui sont offertes aux étudiants, comme une bourse d'études, mais également des services qui peuvent soutenir leur capacité à recourir à celles-ci dans la visée de réaliser le parcours scolaire choisi.

Malgré l'éclairage des résultats de l'analyse typologique, cette dernière comporte des limites. Un fort accent a été mis sur le milieu d'études institutionnel, et pas assez sur les différences de contexte et de taille des universités étudiées, de même que sur l'origine des répondantes. II en résulte qu'il n'est pas évident de nous prononcer clairement pour affirmer si les différences observées entre nos deux cas tiennent aux différences institutionnelles à l'égard des étudiants autochtones, aux différences situationnelles et/ou aux différences entre l'origine des répondantes. II aurait probablement été possible de le faire avec un nombre plus élevé de cas et de participantes. C'est pourquoi nous croyons que cette étude pourrait être reprise dans plusieurs universités avec un plus grand nombre de répondantesÂ provenant, par exemple, de communautés autochtones particulières. Une autre limite est liée aux choix des participantes. II serait judicieux que d'éventuelles recherches s'intéressent aux parcours de femmes autochtones qui ont arrêté leur programme de formation universitaire afin de mettre en lumière les failles des universités dans le soutien apporté à leurs possibilités de choix. Par ailleurs, cette recherche a relevé des pratiques favorables pour soutenir les possibilités de réaliser le parcours scolaire désiré de femmes autochtones. Or, une recherche future pourrait s'inspirer de ces résultats pour démarrer une recherche participative en partenariat avec des universités et des organisations autochtones afin de bonifier l'offre de services existante.

\section{References}

Adelman, H. S., Taylor, L. et Nelson, P. (2010). Native American students going to and staying in post- secondary education. American Indian Culture and Research Journal, 37(3), 29-56.

Alfred, T. (2004). Warrior scholarship. Dans D. A. Mihesuah et A. C. Wilson (dir.), Indigening the academy. Transforming scholarship and empowering communities (p. 88-100). Lincoln, NE : University of Nebraska Press.

Anaya, J. (2014). Report of the special rapporteur on the rights of indigenous peoples. New York, NY : Nations Unies.

Andrade, M. S. (2014). Not what every other girl wants: American Indian women's educational aspirations. Religion \& Education, 41, 290-309.

Aragon, S. R. (2002). An investigation of factors influencing classroom motivation for postsecondary American Indian/Alaska native students. Journal of American Indian Education, 41(1), 1-18.

Arbor, A. (2008). Pathway to the Ph.D.: Experiences of highachieving American Indian females. (Thèse de doctorat, University of Oklahoma, Norman).

Bailey, K. A. (2015). Racism within the Canadian university: Indigenous students' experiences. Ethnic and Racial Studies, 39(7), 1261-1279.

Bamdas, J. A. (2009). Emerging narratives of Native American, Asian American, and African American women in middle adulthood with an education doctorate degree. (Thèse de doctorat, Florida Atlantic University, Boca Raton).

Battiste, M. (2013). Decolonizing education: Nourishing the learning spirit. Saskatoon, Canada : Purich Publishing.

Bonvin, J.-M. (2014). L'approche par les capabilités au service de l'analyse des politiques sociales. Québec, Canada : CRIEVAT.

Castagno, A. E. (2005). Extending the bounds of race and racism: Indigenous women and the persistence of the black-white paradigm of race. The urban review, 37(5), 447-468.

Commission de vérité et réconciliation. (2015). Commission de vérité et réconciliation du Canada : Appels à l'action. Winnipeg, Canada : Commission du Canada. 
Commission royale sur les peuples autochtones. (1996). Rapport de la Commission royale sur les peuples autochtones. Ottawa, Canada : Commission du Canada.

Conseil supérieur de l'éducation. (2016). Rapport sur l'état et les besoins de l'éducation 2014-2016. Remettre le cap sur l'équité. Québec, Canada : Gouvernement du Québec.

Dubet, F. (2010). Les places et les chances. Paris, France : Éditions du Seuil.

Fox, M. J. (2013). American Indian/Alaska Native women: The path to the doctorate. Journal of American Indian Education, 52(1), 26-44.

Guimond, E., Fonda, M., Jetté, D. et Sirois, L. (2012). Les femmes autochtones. Éducation et principaux domaines d'études. Ottawa : Affaires autochtones et Développement du Nord Canada.

Huffman, T. (2001). Resistance theory and the transculturation hypothesis as explanations of college attrition and persistence among culturally traditional American Indian students. Journal of American Indian Education, 40(3), 1-39.

Jaime, A. M. (2003). A room without a view from within the ivory tower. American Indian Quarterly, 27(1-2), 252-263.

Joncas, J.-A. (2013). Apport à la compréhension de l'expérience scolaire de persévérants universitaires des Premières Nations au Québec (Mémoire de maîtrise, Université du Québec à Chicoutimi, Canada).

Joncas, J. et Roy, B. (2016). Les grossesses chez les adolescentes autochtones au Canada : Un portrait critique de la littérature. Recherches amérindiennes au Québec, 45(1), 17-27.

Kerr, J. (2014). Western epistemic dominance and colonial structures. Decolonizing: Indigeneity, Education \& Society, 3(2), 83-104.

Larimore, J. A. et McClellan, G. S. (2005). Native American student retention in U.S. postsecondary education. New Directions for Students Services, $109,17-32$.

Lavell Harvard, D. M. (2011). The power of silence and the price of success: Academic achievement as transformational resistance for aboriginal women. (Thèse de doctorat, The University of Western Ontario, London, Canada).

Lévesque, C. (2015). Promouvoir la sécurisation culturelle pour améliorer la qualité de vie et les conditions de santé de la population autochtone. Revue Droits et Libertés, 34(2), 16-19.

Lévesque, C. et Labrecque, F. (2007). Itinéraires d'égalité. Trajectoires des femmes autochtones du Québec et du Canada. Cahiers DIALOG, 2007(3), 1-130.

Martin, J. V. (2001). Voices from the heart of the circle: Eight Aboriginal women reflect on their experiences at university. (Thèse de doctorat, University of Alberta, Edmonton, Canada).

Montgomery, D., Minville, M. L., Winterowd, C., Jeffries, B. et Baysden, M. F. (2000). American Indian college students: An exploration into resiliency factors revealed through personal stories. Cultural diversity and ethnic minority psychology, 6(4), 387-398.

Mihesuah, J. K. (2004). Graduating indigenous students by confronting the academic environment. Dans $D$.

A. Mihesuah et A. C. Wilson (dir.), Indigening the Academy. Transforming scholarship and empowering communities (p. 191-199). Lincoln, NE : University of Nebraska Press.

Mosholder, R., Waite, B. et Chris, G. (2011). Encouraging post-secondary Native American student persistence. Orem, UT : Utah Valley University.

Neeganagwedgin, E. (2011). A critical review of Aboriginal education in Canada: Eurocentric dominance impact and everyday denial. International Journal of inclusive Education, 17(1), 15-31.

Pidgeon, M., Archibald, J.-A., et Hawkey, C. (2014). Relationships matter: Supporting Aboriginal graduate students in British Columbia, Canada. Canadian Journal of Higher Education, 44(1), 1-21.

Potvin, M. (2014). Diversité ethnique et éducation inclusive : fondements et perspectives. Éducation et sociétés, 1(33), 185-202.

Rasmussen, D. (2001). Qallunology: A Pedagogy for the Oppressor. Canadian Journal of Native Education, 25(2), 105-116. 
Ratel, J.-L. (2017). Des projets d'études aux projets de vie. Une analyse des parcours universitaires chez les étudiants des Premières Nations au Québec. Cahiers du CIÉRA, 14, 84-104.

Rodon, T. (2008). Les étudiants autochtones à l'Université Laval : enquête sur les besoins et les problématiques. Les cahiers du CIÉRA, 1, 5-13.

Sankhulani, L. (2007). Gender issues in aboriginal learning. Saskatoon, Canada : Conseil canadien sur l'apprentissage.

Schnapper, D. (2012). La compréhension sociologique. Démarche de l'analyse typologique (3e éd.). Paris, France : PUF.

Sen, A. (2000). Repenser l'inégalité. Paris, France : Éditions du Seuil.

Sen, A. (2010). L'idée de justice. Paris, France : Flammarion.

Sonn, C., Bishop, B. et Humphries, R. (2000). Encounters with the dominant culture: Voices of indigenous students in mainstream higher education. Australian psychologist, 35(2), 128-135.

Statistique Canada. (2016a). Les femmes des Premières Nations, les Métisses et les Inuites. Ottawa, Canada : Statistique Canada.

Statistique Canada. (2016b). Recensement de 2016. Ottawa, Canada : Gouvernement du Canada.

Timmons, V. (2009). Retention of aboriginal students in post-secondary institutions in Atlantic Canada. Halifax, Canada : Association of Atlantic Universities.

Verhoeven, M., Orianne, J.-F. et Dupriez, V. (2007). Vers des politiques d'éducation " capacitantes "? Formation emploi, 98(Numéro spécial), 93-105.

Waterman, S. J. et Lindley, L. S. (2013). Cultural strengths to persevere: Native American women in higher education. Journal about Women in Higher Education, 6(2), 139-165.

Whitley, J. (2014). Supporting educational success for aboriginal students: identifying key influences. McGill Journal of Education, 49(1), 155-182.

Yin, R. K. (2012). Applications of case study research. Thousand Oaks, CA: Sage.

\section{Contact Information}

Jo-Anni Joncas

jjoncas2@uottawa.ca

\section{Notes}

1 Nous utilisons les termes "Autochtones " et " Premiers peuples " pour désigner les personnes et les peuples étant les premiers habitants des terres nommées Canada qui ont résisté et résistent encore au colonialisme de peuplement, incluant tant les Premières Nations que les Inuits et les Métis. 